\title{
ON GENERALISED LAH-NUMBERS
}

\author{
by SELMO TAUBBER
}

(Received 11th May 1964)

\section{Introduction}

In (1) numbers related to the Stirling numbers are defined. Later in (2) these numbers were called Lah-numbers (cf. 2, p. 43, Ex. 16). According to (1) these numbers are of importance in Mathematical Statistics. In this paper we shall generalise the method and apply it to generalised Stirling numbers as defined in (3).

In (3) the polynomials $Q$ and the numbers $A_{n}^{s}$ and $B_{n}^{s}$ were defined by the relations

$$
\begin{aligned}
Q(x ; M, N, n)=Q(x, n)= & \prod_{m=1}^{n}[M(m)+N(m) x]=\sum_{s=0}^{n} A_{n}^{s} x^{s}, n \geqq 1, \\
& Q(x, 0)=M(0), \\
x^{n}= & \sum_{m=0}^{n} B_{n}^{m} Q(x, m), \\
1= & B_{0}^{0} Q(x, 0)=B_{0}^{0} M(0),
\end{aligned}
$$

where $M$ and $N$ are two functions such that $M(0) \neq 0, M(n)$ is defined for all positive integers $n$, and $N(n)$ is defined for all positive integers $n$ and $N(n) \neq 0$. The numbers $A_{n}^{s}$ and $B_{n}^{s}$ are called generalised Stirling numbers.

\section{Relationship between different $\mathbf{Q}$-polynomials}

Let $Q_{1}(x, n)=Q\left(x ; M_{1}, N_{1}, n\right)$ and $Q_{2}(x, n)=Q\left(x ; M_{2}, N_{2}, n\right)$, then

and

$$
\begin{aligned}
& Q_{1}(x, n)=\sum_{s=0}^{n} A_{1, n}^{s} x^{s}, \quad x^{n}=\sum_{m=0}^{n} B_{1, n}^{m} Q_{1}(x, m), \\
& Q_{1}(x, 0)=M_{1}(0), \quad 1=B_{1,0}^{0} Q_{1}(x, 0)=B_{1,0}^{0} M_{1}(0),
\end{aligned}
$$

$$
\begin{gathered}
Q_{2}(x, n)=\sum_{s=0}^{n} A_{2, n}^{s} x^{s}, \quad x^{n}=\sum_{m=0}^{n} B_{2, n}^{m} Q_{2}(x, m), \\
Q_{2}(x, 0)=M_{2}(0), \quad 1=B_{2,0}^{0} Q_{2}(x, 0)=B_{2,0}^{0} M_{2}(0),
\end{gathered}
$$

where $A_{n}^{m}$ and $B_{n}^{m}$ are zero for $m>n, m<0, n<0$.

Let us express $Q_{2}(x, n)$ in terms of $Q_{1}(x, m)$. We can write

$$
\begin{gathered}
Q_{2}(x, n)=\sum_{s=0}^{n} A_{2, n}^{s} x^{s}=\sum_{s=0}^{n} A_{2, n}^{s}\left[\sum_{m=0}^{s} B_{1, s}^{m} Q_{1}(x, m)\right], \\
Q_{2}(x, n)=\sum_{s=0}^{n} \sum_{m=0}^{s} A_{2, n}^{s} B_{1, s}^{m} Q_{1}(x, m) .
\end{gathered}
$$


Considering the conditions on the numbers $A$ and $B$ we can extend the second summation to $n$ and then change the order of summation, thus,

$$
Q_{2}(x, n)=\sum_{s=0}^{n} \sum_{m=0}^{n} A_{2, n}^{s} B_{1, s}^{m} Q_{1}(x, m)=\sum_{m=0}^{n} Q_{1}(x, m) \sum_{s=0}^{n} A_{2, n}^{s} B_{1, s}^{m},
$$

and, using again the conditions on the $A$ and $B$ numbers, we have,

Let

$$
Q_{2}(x, n)=\sum_{m=0}^{n} Q_{1}(x, m) \sum_{s=m}^{n} A_{2, n}^{s} B_{1, s}^{m}
$$

$$
\sum_{s=m}^{n} A_{2, \mathrm{n}}^{s} B_{1, \mathrm{~s}}^{m}=L_{2, \mathrm{l}, \mathrm{n}}^{m}
$$

where $L_{2,1, n}^{m}=0$ for $m<0, n<0, m>n$.

Similarly, if we express $Q_{1}(x, n)$ in terms of $Q_{2}$-polynomials we obtain

with

$$
Q_{1}(x, n)=\sum_{m=0}^{n} Q_{2}(x, m) \sum_{s=m}^{n} A_{1, n}^{s} B_{2, s}^{m},
$$

$$
\sum_{s=m}^{n} A_{1, n}^{s} B_{2, s}^{m}=L_{1,2, n}^{m}
$$

and where $L_{1,2, n}^{m}=0$, for $n<0, m<0, n<m$. It is clear that in the special case where $Q_{1}=Q_{2}, L_{1,2, n}^{m}=L_{2,1, n}^{m}=\delta_{n}^{m}$, where $\delta_{n}^{m}$ is the Kronecker delta.

\section{Quasi-orthogonality of the L-numbers}

For obvious reasons we shall call the $L$-numbers generalised Lah-numbers (cf. Ex. 2 hereafter). Using (7) and (8) we can write

and using (9) and (10)

$$
Q_{2}(x, n)=\sum_{m=0}^{n} Q_{1}(x, m) L_{2,1, n}^{m}
$$

$$
Q_{1}(x, n)=\sum_{m=0}^{n} Q_{2}(x, m) L_{1,2, n}^{m}
$$

Substituting (12) into (11) we obtain

$$
Q_{2}(x, n)=\sum_{m=0}^{n} L_{2,1, n}^{m} \sum_{s=0}^{n} L_{1,2, m}^{s} Q_{2}(x, s),
$$

and using the conditions on the $L$-numbers we obtain

thus

$$
Q_{2}(x, n)=\sum_{s=0}^{n} Q_{2}(x, s) \sum_{m=s}^{n} L_{2,1, n}^{m} L_{1,2, m}^{s}
$$

$$
\sum_{m=s}^{n} L_{2,1, n}^{m} L_{1,2, m}^{s}=\delta_{n}^{s} \text {. }
$$

(13) expresses the quasi-orthogonality of $L$-numbers for reversed indices. 


\section{Recurrence relations}

According to (1) we can write and,

$$
Q_{1}(x, n+1)=\sum_{m=0}^{n+1} A_{1, n+1}^{m} x^{m}=\left[M_{1}(n+1)+N_{1}(n+1) x\right] \sum_{m=0}^{n} A_{1, n}^{m} x^{m},
$$

$$
Q_{2}(x, n+1)=\sum_{m=0}^{n+1} A_{2, n+1}^{m} x^{m}=\left[M_{2}(n+1)+N_{2}(n+1) x\right] \sum_{m=0}^{n} A_{2, n}^{m} x^{m},
$$

which according to (11) yields

$$
\begin{array}{r}
Q_{2}(x, n+1)=\sum_{m=0}^{n+1} Q_{1}(x, m) L_{2,1, n+1}^{m}=\left[M_{2}(n+1)+N_{2}(n+1) x\right] \\
\sum_{m=0}^{n} Q_{1}(x, m) L_{2,1, n}^{m} .
\end{array}
$$

But

$$
Q_{1}(x, m+1)=\left[M_{1}(m+1)+N_{1}(m+1) x\right] Q_{1}(x, m) \text {, }
$$

so that

$$
x Q_{1}(x, m)=\left[Q_{1}(x, m+1)-M_{1}(m+1) Q_{1}(x, m)\right] / N_{1}(m+1) .
$$

Substituting (15) into (14) we obtain

$$
\begin{aligned}
& \sum_{m=0}^{n+1} Q_{1}(x, m) L_{2,1, n+1}^{m}=M_{2}(n+1) \sum_{m=0}^{n} Q_{1}(x, m) L_{2,1, n}^{m} \\
& \quad+N_{2}(n+1) \sum_{m=0}^{n}\left[Q_{1}(x, m+1)-M_{1}(m+1) Q_{1}(x, m)\right] L_{2,1, n}^{m} / N_{1}(m+1) .
\end{aligned}
$$

By equating the coefficients of $Q_{1}(x, m)$ we obtain or,

$$
L_{2,1, n+1}^{m}=M_{2}(n+1) L_{2,1, n}^{m}+\frac{N_{2}(n+1)}{N_{1}(m)} L_{2,1, n}^{m-1}-\frac{N_{2}(n+1) M_{1}(m+1)}{N_{1}(m+1)} L_{2,1, n}^{m},
$$

$$
L_{2,1, n}^{m}=\left[M_{2}(n)-\frac{N_{2}(n) M_{1}(m+1)}{N_{1}(m+1)}\right] L_{2,1, n-1}^{m}+\frac{N_{2}(n)}{N_{1}(m)} L_{2,1, n-1}^{m-1}
$$

and by inverting the indices 2 and 1 ,

$$
L_{1,2, n}^{m}=\left[M_{1}(n)-\frac{N_{1}(n) M_{2}(m+1)}{N_{2}(m+1)}\right] L_{1,2, n-1}^{m}+\frac{N_{1}(n)}{N_{2}(m)} L_{1,2, n-1}^{m-1} .
$$

\section{Examples}

1. Let $M_{1}(\alpha)=\alpha, N_{1}(\alpha)=\alpha-1, M_{2}(\alpha)=\alpha+1, N_{2}(\alpha)=1 / \alpha$. We obtain, taking $L_{2,1,1}^{1}=L_{1,2,1}^{1}=1$, and $\alpha>0$,

$$
\begin{aligned}
& L_{2,1, n}^{m}=\left[(n+1)-\frac{m+1}{n m}\right] L_{2,1, n-1}^{m}+\frac{1}{n(m-1)} L_{2,1, n-1,}^{m-1}, \\
& L_{1,2, n}^{m}=[n-(n-1)(m+1)(m+2)] L_{1,2, n-1}^{m}+m(n-1) L_{1,2, n-1}^{m-1},
\end{aligned}
$$


and the following numerical values:

\begin{tabular}{rrrrrr}
$L_{2,1, n}^{m}:$ & $m=$ & & & 3 & 4 \\
& $n:$ & & & & \\
1 & 1 & & & \\
2 & 2 & $1 / 2$ & & \\
3 & $20 / 3$ & $29 / 12$ & $1 / 12$ & \\
$L_{1,2, n}^{m}:$ & $m=$ & 1 & 2 & 3 & 4 \\
& $n:$ & & & & \\
1 & 1 & & & \\
2 & -4 & 2 & & \\
3 & 36 & -58 & $1233 / 96$ & $199 / 288$ & $1 / 144$ \\
4 & -504 & 2072 & -1194 & 144 \\
\hline
\end{tabular}

2. Let $M_{1}(\alpha)=1-\alpha, N_{1}(\alpha)=1, M_{2}(\alpha)=\alpha-1, N_{2}(\alpha)=1$, then the numbers obtained are the Lah-numbers as studied in (1).

\section{REFERENCES}

(1) I. LAH, Eine neue Art von Zahlen, ihre Eigenschaften und Anwendungen in der mathematischen Statistik, Mitteilungsblatt fuer Mathematische Statistik, 7 (1955), 203-212.

(2) J. Riordan, An Introduction to Combinatorial Analysis (New York, 1958).

(3) S. TAUBer, On quasi-orthogonal numbers, Amer. Math. Monthly, 69 (1962), 365-372.

Portland State College

Portland

OREGON 